\title{
DEPOSIÇÃO DA APLICAÇÃO DE HERBICIDAS E FERTILIZANTE FOLIAR NO MANEJO DE PLANTAS DANINHAS NA CULTURA DO MILHO
}

\author{
CLEBER DANIEL DE GOES MACIEL ${ }^{1}$, ENELISE OSCO HELVIG ${ }^{1}$, \\ ANDRÉ AUGUSTO PAZINATO DA SILVA', JOÃO PAULO MATIAS ${ }^{1}$, \\ JOSÉ CRISTIMIANO DOS SANTOS NETO ${ }^{1}$ e DÉCIO KARAM ${ }^{2}$
}

\begin{abstract}
${ }^{1}$ Universidade Estadualdo Centro-Oeste-UNICENTRO,cmaciel@unicentro.br, ene_oso@hotmail.com, andrepazinato0@gmail.com,jpmatias2@gmail.com,neto.buri@hotmail.com

${ }^{2}$ Embrapa Milho e Sorgo, 35701-970, Sete Lagoas-MG, decio.karam@embrapa.br
\end{abstract}

Revista Brasileira de Milho e Sorgo, v.18, n.3, p. 364-378, 2019

\begin{abstract}
RESUMO - O trabalho teve como objetivo avaliar a deposição da pulverização, assim como a seletividade e eficiência no controle de plantas daninhas de misturas em tanque de herbicidas associadas ou não ao fertilizante foliar Fertiactyl $\mathrm{Pós}^{\circledR}$ na cultura do milho. Dois experimentos foram realizados em campo com os híbridos P2530 ${ }^{\circledR}$ (convencional) e DKB 330 PRO $2^{\circledR}$ (transgênico), na fazenda escola da Universidade Estadual do Centro-Oeste - UNICENTRO, Campus CEDETEG, Guarapuava-PR, durante a safra 2016/2017. O delineamento utilizado foi o de blocos ao acaso, com oito tratamentos e cinco repetições, em fatorial $3 \times 2+2$. Os fatores foram três misturas de herbicidas com atrazine (ATR) + nicosulfuron (NIC) $\left(1000+45\right.$ g i.a. ha $\left.{ }^{-1}\right)$, ATR + mesotrione (MES) $\left(1000+144\right.$ g i.a. ha $\left.{ }^{-1}\right)$; ATR + tembotrione (TEM) $\left(1000+100,8\right.$ g i.a. ha $\left.{ }^{-1}\right)$, duas condições de associação ou não ao fertilizante foliar Fertiactyl Pós ${ }^{\circledR}$ (FEP) (400 $\mathrm{mL} \mathrm{ha}^{-1}$ ), com testemunhas capinadas e sem capina. A associação de FEP com os herbicidas ATR + NIC, ATR + MES e ATR + TEM não interferiu na deposição da pulverização em milho e erva-quente (Spermacoce latifolia), assim como na eficiência de controle de plantas daninhas e produtividade de grãos. O FEP associado às misturas em tanque dos herbicidas apenas apresentou resultado promissor na redução da fitointoxicação visual e manutenção dos níveis de clorofila iniciais na cultura do milho.
\end{abstract}

Palavras-chave: Fitointoxicação; mistura em tanque; Fertiactyl Pós ${ }^{\circledR}$, Zea mays L.

\section{DEPOSITION OF HERBICIDE AND FOLIAR FERTILIZER ON WEED MANAGEMENT IN MAIZE CROP}

\begin{abstract}
The aim of this work was to evaluate the dynamic of spray deposition as well as the selectivity and efficiency in the weed control of herbicides mixtures in tank, associated or not with Fertiactyl Pós ${ }^{\mathrm{TM}}$ leaf fertilizer in maize crop. Two experiments were carried out at field with $\mathrm{P} 2530^{\mathrm{TM}}$ and DKB $330 \mathrm{PRO} 2^{\mathrm{TM}}$ hybrids, in the school farm of the Universidade Estadual do Centro-Oeste - UNICENTRO, Campus CEDETEG, Guarapuava - PR/Brazil, during the 2016/2017 harvest seasons. The experimental layout was a completely randomized block design, with eight treatments and five replications, in a factorial scheme $3 \times 2+2$. The factors were three herbicides mixtures $\left(\mathrm{g}\right.$ ai ha $\left.\mathrm{h}^{-1}\right)+\left(\mathrm{mL} \mathrm{pc} \mathrm{ha}^{-1}\right)$, with two conditions of association with the Fertiactyl Pós ${ }^{\mathrm{TM}}$ leaf fertilizer: atrazine (ATR) + nicosulfuron (NIC) (1000 + 45); ATR + mesotrione (MES) $(1000+144) ;$ ATR + tembotrione (TEM) $(1000+100.8) ;$ ATR + NIC + Fertiactyl Pós $^{\text {TM }}($ FEP $)(1000+45)+(400) ;$ ATR + MES + FEP $(1000+144)+(400) ;$ ATR + TEM + FEP $(1000+100.8)+(400)$; and controls with and without weeding. The FEP association with the herbicides ATR + NIC, ATR + MES and ATR + TEM did not affect the spray deposition in plants of maize and Spermacoce latifolia, as well as the weed control efficiency and grain yield. The FEP associated with herbicides mixtures in tank can be used to reduce phytotoxicity and to maintain the initial levels of chlorophyll in maize crop.
\end{abstract}

Keywords: Phytointoxication, tank mixture, Fertiactyl Pós ${ }^{\mathrm{TM}}$, Zea mays L. 
A cultura do cereal milho está entre as principais cultivadas no mundo, principalmente em razão da sua quantidade e qualidade de reservas acumuladas nos grãos, fornecendo produtos para utilização na alimentação humana, animal e matérias-primas para a indústria (Alves et al., 2015).

O Brasil apresenta grande potencial para o cultivo do milho, porém, o clima tropical favorece a ocorrência de elevada quantidade de plantas daninhas, as quais interferem no desenvolvimento e na produtividade da cultura (Carvalho et al., 2010). Portanto, é necessário que se realize correto manejo de plantas daninhas, neste sentido, as perdas de produto na aplicação podem gerar falhas de controle, bem como seleção de indivíduos resistentes a herbicidas, além de contaminações ambientais (Terra et al., 2014).

Por causa do problema de fitointoxicação em plantas de milho, causado por herbicidas, estudos que desenvolvam alternativas para amenizar os danos às plantas cultivadas são necessários. Por exemplo, o uso de fertilizantes foliares com ação bioestimulante. De acordo com Calvo et al. (2014), os bioestimulantes são substâncias ou microrganismos usados para potencializar o crescimento das plantas, geralmente aumentando a habilidade de assimilar os nutrientes aplicados ou promover benefícios em relação ao desenvolvimento. A definição de bioestimulantes de plantas ainda está em evolução, sendo em parte devida ao reflexo da diversidade de insumos que podem ser considerados estimulantes.

Os diferentes bioestimulantes têm potencial para aumento de biomassa das plantas, produtividade e resistência a múltiplos tipos de estresse (Calvo et al., 2014; Nardi et al., 2016). Porém, pouca ênfase tem sido dada à hipótese de que os bioestimulantes também podem prevenir a toxicidade de herbicidas em espécies cultivadas (Constantin et al., 2016), sem prejudicar o controle das plantas daninhas.

A aplicação de herbicidas representa uma forma de estresse abiótico nas plantas de forma geral, por causa de efeitos secundários relacionados à metabolização nos tecidos vegetais e à recuperação de lesões fisiológicas. Nesse sentido, o fertilizante foliar Fertiactyl Pós ${ }^{\circledR}$ é um produto que contém um complexo de bioestimulantes eficientes na absorção de nutrientes, assim como no estímulo da atividade fisiológica para resistência ao estresse, desenvolvimento radicular e aumento da fotossíntese (Santos et al., 2015; Constantin et al., 2016).

Portanto, o trabalho teve como objetivo avaliar a dinâmica de deposição da pulverização, assim como a seletividade e eficiência de controle de plantas daninhas na cultura do milho, utilizando misturas em tanque de herbicidas associadas ou não ao fertilizante foliar Fertiactyl Pós ${ }^{\circledR}$.

\section{Material e Métodos}

Dois experimentos foram conduzidos em campo na fazenda escola da Universidade Estadual do Centro-Oeste - UNICENTRO, localizada em Guarapuava-PR, sob as coordenadas geográficas S $25^{\circ} 22^{\prime} 56,0^{\prime \prime}, \mathrm{W} 051^{\circ} 33^{\prime} 16,7^{\prime \prime}$ e a $986 \mathrm{~m}$, durante a safra $2016 / 2017$.

O clima da região é classificado por KöeppenGeiger como $\mathrm{Cfb}$ subtropical mesotérmico úmido (Peel et al., 2007), com verões frescos, invernos com ocorrência de geadas severas e frequentes, não apresentando estação seca. As temperaturas médias anuais variam de $16^{\circ} \mathrm{C} \mathrm{a} 17^{\circ} \mathrm{C}$ e a precipitação média anual em torno de $1.500 \mathrm{~mm}$. Os dados meteorológicos diários de temperatura (máximas e mínimas) e pluviometria registrados durante a condução dos experimentos encontram-se dispostos na Figura 1, os quais 
foram obtidos na estação experimental localizada na Universidade Estadual do Centro-Oeste.

O solo da área experimental é classificado como Latossolo Bruno distrófico típico, de textura argilosa (Santos et al., 2013a), constituído por teores de argila, areia e silte de 560, 180 e $260 \mathrm{~g} \mathrm{~kg}^{-1}$, respectivamente. $\mathrm{Na}$ semeadura, o solo apresentava as seguintes características químicas: $\mathrm{pH}$ em $\mathrm{CaCl}_{2}$ de 4,77; $\mathrm{H}+\mathrm{Al}^{+3}$ de 4,96 $\mathrm{cmol}_{\mathrm{c}} \mathrm{dm}^{-3} ; \mathrm{Ca}^{+2}$ de 2,81 $\mathrm{cmol}_{\mathrm{c}}$ $\mathrm{dm}^{-3} ; \mathrm{Mg}^{+2}$ de $1,49 \mathrm{cmol}_{\mathrm{c}} \mathrm{dm}^{-3} ; \mathrm{K}^{+}$de $0,31 \mathrm{cmol}_{\mathrm{c}} \mathrm{dm}^{-3}$; $\mathrm{P}$ de 2,68 $\mathrm{mg} \mathrm{dm}^{-3}$ (Mehlich) e C $27 \mathrm{~g} \mathrm{dm}^{-3}$.

Os experimentos foram instalados em área contendo inicialmente a aveia-preta cultivar IAPAR 61 como cobertura do solo. A dessecação da aveia-preta foi realizada no estádio reprodutivo com $960 \mathrm{~g} \mathrm{ha}^{-1}$ de glyphosate, e posterior roçagem do material dessecado, seguida de escarificação e gradagens do solo, assim como de incorporação dos resíduos culturais.
A semeadura dos experimentos foi realizada em 16/11/2016, utilizando os híbridos $\mathrm{P} 2530^{\circledR}$ (convencional) e DKB 330PRO2 ${ }^{\circledR}$ (transgênico), em espaçamento entre linhas de $0,8 \mathrm{~m}$, população de 62.500 plantas ha-1 e adubação de base de $300 \mathrm{~kg} \mathrm{ha}^{-1}$ do formulado 08-20-20 (NPK). Em complemento, na adubação de cobertura utilizaram-se $80 \mathrm{~kg} \mathrm{ha}^{-1}$ de ureia $(45 \% \mathrm{~N})$, no estádio $\mathrm{V}_{3}-\mathrm{V}_{4}$ da cultura. Essa aplicação foi realizada superficialmente a lanço (sem incorporação), sob boas condições de umidade do solo.

O delineamento utilizado foi o de blocos ao acaso, com oito tratamentos e cinco repetições, em esquema fatorial $3 \times 2+2$. Os fatores foram representados por três misturas em tanque de herbicidas com atrazine (ATR) + nicosulfuron (NIC) $(1000+45 \mathrm{~g}$ i.a. ha-1 ${ }^{-1}$, ATR + mesotrione (MES) $(1000+144 \mathrm{~g}$ i.a. ha $\left.{ }^{-1}\right) ;$ ATR + tembotrione (TEM) $(1000+100,8$ g i.a. ha $\left.{ }^{-1}\right)$, duas condições de associação ou não ao

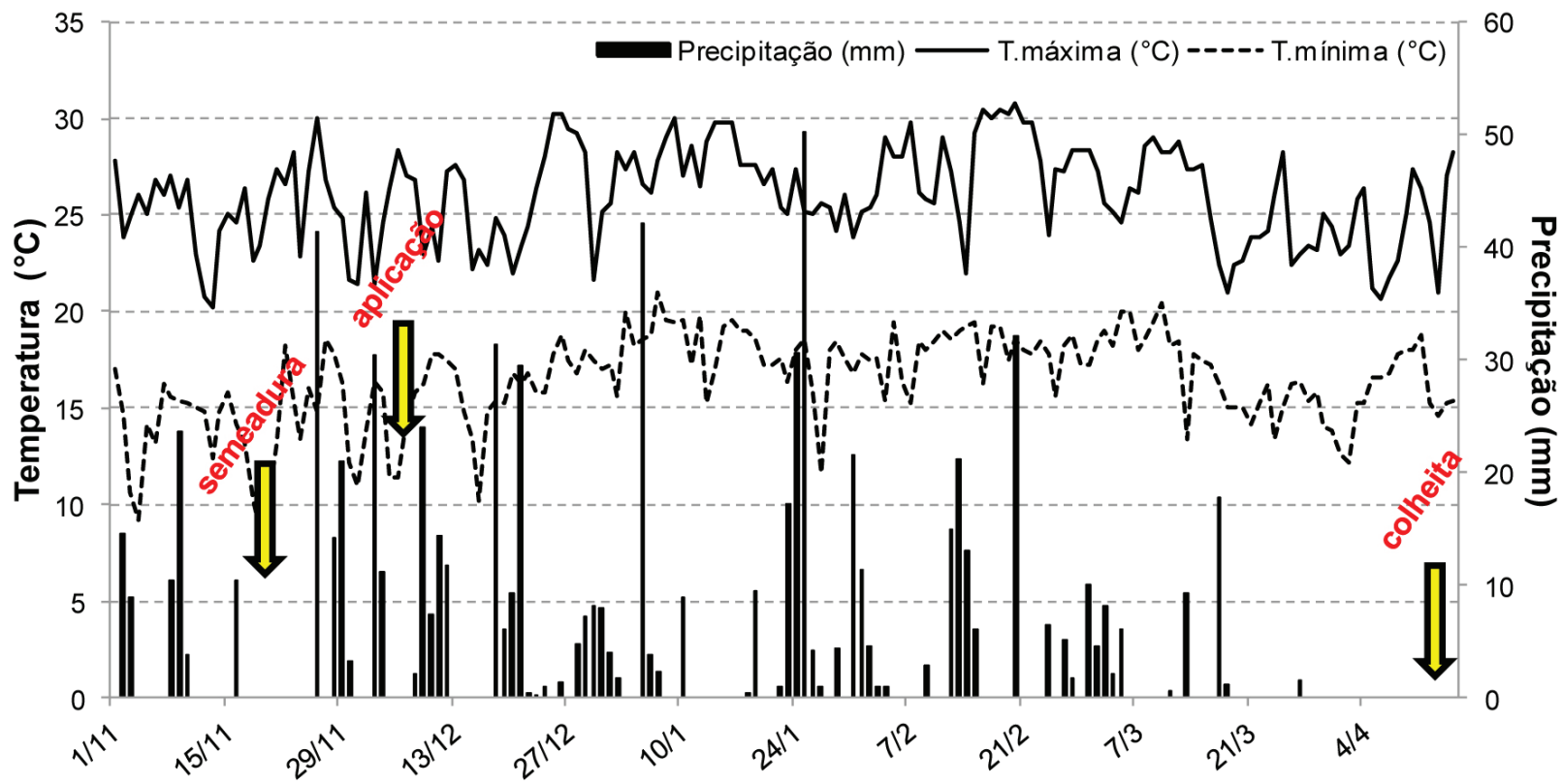

Safra 2016/17

Figura 1. Dados meteorológicos diários de temperatura $\left({ }^{\circ} \mathrm{C}\right)$ e precipitação $(\mathrm{mm})$ registrados durante a condução da cultura do milho. Guarapuava-PR, Safra 2016/17. 
fertilizante foliar Fertiactyl Pós ${ }^{\circledR}$ (FEP) $\left(400 \mathrm{~mL} \mathrm{ha}^{-1}\right)$, com testemunhas capinadas e sem capina. Em todos os tratamentos adicionaram-se os adjuvantes Desade$\operatorname{re}^{\circledR}(0,1 \% \mathrm{v} / \mathrm{v})+$ AllerBiW $^{\circledR}(0,05 \% \mathrm{v} / \mathrm{v})$. As unidades experimentais constituíram-se de parcelas com área total de 3,5 x 4,0 $\mathrm{m}\left(14,0 \mathrm{~m}^{2}\right)$, contendo quatro linhas para cada híbrido de base genética contrastante, ambos em sistema de semeadura convencional. Nas avaliações, utilizaram-se como área útil apenas as linhas centrais das parcelas, desconsiderando $0,5 \mathrm{~m}$ em cada extremidade.

A aplicação dos tratamentos com as misturas em tanque ocorreu em 12/12/2016, sendo realizada com um pulverizador costal pressurizado a $\mathrm{CO}_{2}$, equipado com seis pontas TTi 110.15 (fabricante Teejet ${ }^{\circledR}$ ), espaçadas entre si em $0,5 \mathrm{~m}$ e a $0,5 \mathrm{~m}$ de altura da parte aérea das plantas, e constituindo taxa de aplicação de $150 \mathrm{~L} \mathrm{ha}^{-1}$. As condições meteorológicas no momento da aplicação (início às 11 h e término às 11h50) foram monitoradas com um termo-higro-anemômetro digital portátil. Em média, foram registradas, no início e final das aplicações, temperaturas de $23{ }^{\circ} \mathrm{C}$ e 25 ${ }^{\circ} \mathrm{C}$, umidade relativa de $66 \%$ e $65 \%$, e velocidade dos ventos de 1,5 e 1,2 $\mathrm{km} \mathrm{h}^{-1}$, respectivamente.

Durante todo o ciclo da cultura, o tratamento testemunha capinada foi periodicamente mantido livre da convivência das plantas daninhas, ao contrário da testemunha sem capina, a qual não recebeu nenhuma prática de controle da infestação. As aplicações de fungicidas e inseticidas foram realizadas de maneira preventiva e curativa quando necessário, adotando produtos e doses de acordo com as recomendações técnicas para a cultura do milho.

Para estimativa da deposição da aplicação foi utilizado o traçador azul brilhante (FDC\&1, 1500 ppm) em todas as soluções com as misturas em tanque de herbicidas (Tabela 1). Os alvos foram repre- sentados por folhas dos dois híbridos de milho, os quais no momento da aplicação apresentavam cinco folhas completamente expandidas (estádio $\mathrm{V}_{5}$ ), assim como de uma espécie de planta daninha erva-quente (erva-quente, Spermacoce latifolia) com 3 a 4 folhas (12 plantas $\mathrm{m}^{-2}$ ). Na avaliação de deposição da calda de pulverização foram utilizadas 10 repetições para cada alvo, e o procedimento de recuperação das soluções traçadoras nas folhas dos materiais coletados constituiu-se da embalagem das amostras separada em sacos plásticos e do encaminhamento ao laboratório para lavagem dos alvos plantas de milho e plantas daninhas, com 60 e $25 \mathrm{~mL}$ de água destilada, respectivamente.

Na quantificação do traçador azul brilhante foi utilizado um espectrofotômetro, com resultados da leitura das amostras em absorbância, no comprimento de onda de $630 \mathrm{~nm}$, que proporcioou a transformação em mg L-1, de acordo com coeficiente angular da curva-padrão, e metodologia descrita por Maciel et al. (2011). Os valores de depósitos foram posteriormente transformados em $\mu \mathrm{L} g$ de matéria seca ${ }^{-1}$, sendo as folhas secas em estufa de circulação forçada de ar, por período de 72 horas a $65^{\circ} \mathrm{C}$, após procedimento de lavagem.

As características avaliadas nos híbridos de milho foram fitointoxicação (\%) da cultura e controle de plantas daninhas guanxuma (Sida rhombifolia) e erva-quente (S. latifolia) aos 7, 14, 21 e 28 dias após aplicação (DAA), por meio de escala de notas visuais segundo critérios da Sociedade Brasileira da Ciência das Plantas Daninhas (Velini et al., 1995), em que $0 \%$ correspondeu à ausência de injúria e 100\% à morte das plantas; o teor de clorofila das folhas de milho, com auxílio de clorofilômetro portátil, modelo Minolta SPAD-502 (índice SPAD), utilizando oito plantas por amostra aos 7, 14, 21 e 28 DAA; a altura 
das plantas e espigas, considerando a distância entre o solo até a inserção da última folha e da inserção da primeira espiga, respectivamente, no final do ciclo da cultura.

A colheita foi realizada em 17 de abril de 2017, sendo determinados os componentes de produção: número de grãos por fileira nas espigas (NGF); número de fileiras de grãos das espigas (NF), massa de 100 grãos (P100G) e produtividade de grãos (PROD - $\mathrm{kg} \mathrm{ha}^{-1}$ ), com correção da umidade para $13 \%$. Os resultados de deposição da pulverização foram demonstrados com intervalos de confiança para diferença entre as médias (IC 95\%), a 95\% de probabilidade. As demais variáveis foram submetidas a análise pelo teste $\mathrm{F}$, sendo as médias comparadas pelo teste de Tukey $(\mathrm{p} \leq 0,05)$.

\section{Resultados e Discussão}

Para a deposição das soluções aplicadas na cultura do milho não foram observadas diferenças significativas entre os tratamentos, independentemente da solução, adição de Fertiactyl Pós ${ }^{\circledR}$ (FEP) e híbrido avaliado (Figura 2). Esse mesmo comportamento também ocorreu para a planta daninha erva-quente (S. latifolia). Portanto, pode-se constatar que o FEP, quando associado na calda de pulverização em mistura em tanque com os herbicidas atrazine + nicosulfuron $($ ATR + NIC), atrazine + mesotrione $(\mathrm{ATR}+\mathrm{MES})$ e atrazine + tembotrione (ATR + TEM), não interfere na dinâmica de deposição da calda aplicada nos alvos estudados.

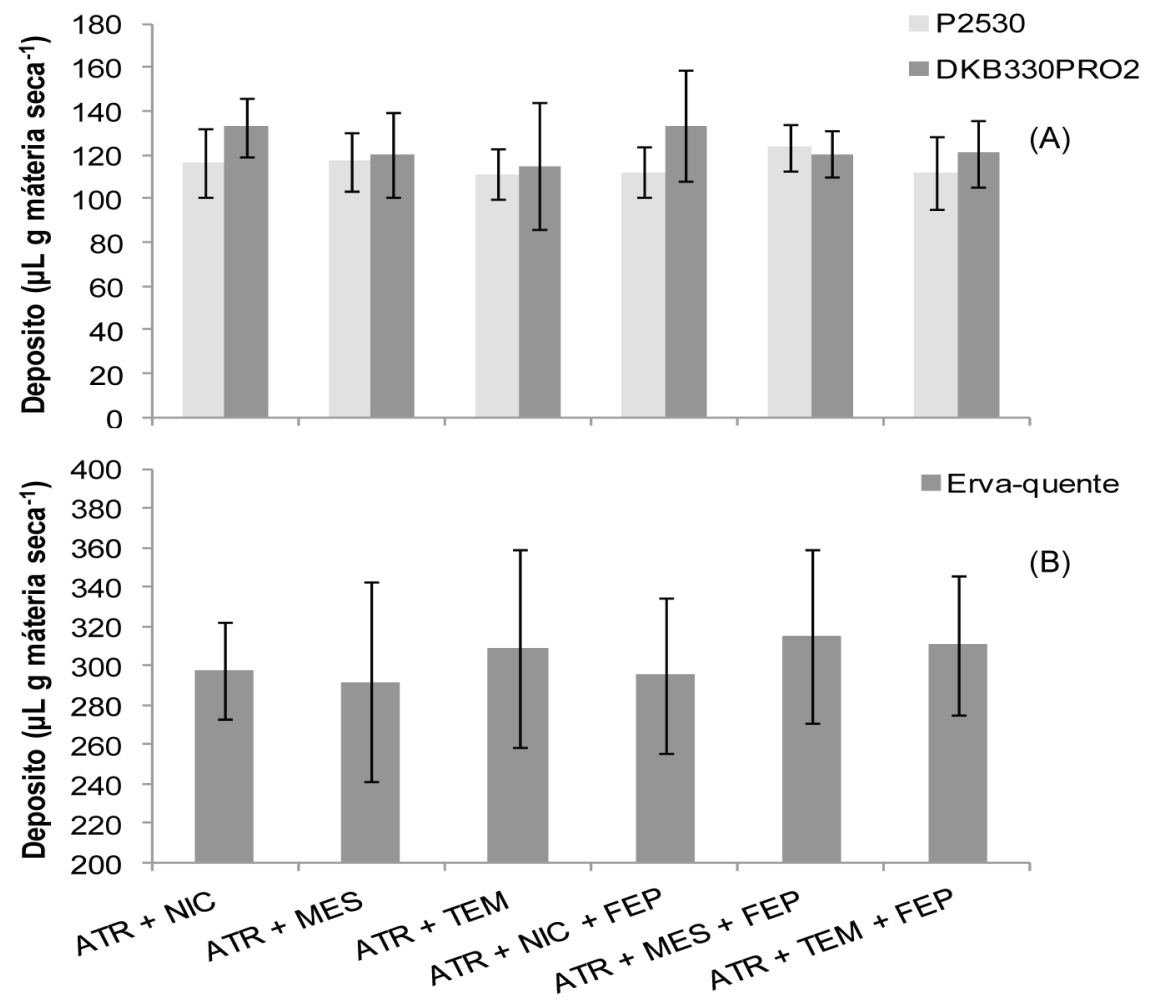

Figura 2. Deposição média da aplicação das misturas em tanque entre os herbicidas atrazine (ATR), nicosulfuron (NIC), mesotrione (MES) e tembotrione (TEM) associadas ou não ao fertilizante foliar Fertiactyl Pós ${ }^{\circledR}$ (FEP) sobre plantas dos híbridos de milho P2530 ${ }^{\circledR}$ e DKB $330 \mathrm{PRO}^{\circledR}$ (A) e planta daninha erva-quente (B). Guarapuava-PR, 2017. 
Em todas as misturas em tanque e épocas avaliadas ocorreram sintomas de fitointoxicação da parte aérea dos híbridos de milho, identificados como clorose nas folhas, sendo constatados os danos mais severos aos 7 dias após a aplicação (DAA) para as associações de herbicidas sem adição de FEP nas caldas de pulverização (Tabela 1). Nessa primeira avaliação, o híbrido DKB $330 \mathrm{PRO}^{\circledR}$ se destacou apenas por ter sido mais sensível aos efeitos fitotóxicos da mistura atrazine + nicosulfuron (ATR + NIC). De forma semelhante, Portugal (2013) relatou que ATR + NIC $\left(1500+50 \mathrm{~g} \mathrm{ha}^{-1}\right)$ também proporcionou apenas leve fitotoxidez ao híbrido VT PRO 1 aos 7 DAA, mas em níveis superiores aos ocorridos com as misturas de ATR + TEM $\left(1500+100,8 \mathrm{~g} \mathrm{ha}^{-1}\right)$ e ATR + MES $\left(1500+120 \mathrm{~g} \mathrm{ha}^{-1}\right)$. Entretanto, todas as associações dos herbicidas com FEP reduziram significativamente o amarelecimento clorótico na parte aérea dos híbridos de milho (Tabela 1), constituindo-se assim uma ferramenta alternativa para minimizar esses danos visualizados nas plantas.

Apesar de a fitointoxicação ter sido progressivamente recuperada ao longo do desenvolvimento inicial da cultura, os resultados mais expressivos da recuperação dos efeitos deletérios ocorreram para misturas de herbicidas com FEP, as quais em média reduziram os sintomas fitotóxicos em 32,0\%, 49,9\% e $70,2 \%$, respectivamente, aos 7, 14 e 21 DAA (Tabela 2). Aos 28 DAA não foram mais constatados sintomas de injúrias para todos os tratamentos.

$\mathrm{Na}$ cultura da soja $\mathrm{RR}^{\circledR}$, Constantin et al. (2016) constataram que a associação do FEP (400 mL $\mathrm{ha}^{-1}$ ) com glyphosate previne perdas de produtividade causadas pela aplicação única ou sequencial desse herbicida. De forma contrária, os autores mencionam que a aplicação de FEP não foi suficiente para amenizar as injúrias causadas à cultura quando associado às misturas de glyphosate + lactofen ou glyphosate + chlorimuron-ethyl. Já Santos et al. (2015) relataram que FEP em mistura em tanque com glyphosate + lactofen foi eficiente em reduzir o estresse fitotóxico, hídrico e incrementar a produtividade da soja MSOY $9144^{\circledR}$. Já em eucalipto, Machado et al. (20171a, 2017b) descreveram FEP + glyphosate como eficiente na supressão da intoxicação e demais efeitos deletérios causados com até $720 \mathrm{~g} \mathrm{ha}^{-1}$ do herbicida, mas também evidenciaram que doses superiores a esta podem prejudicar a cultura.

Para o teor de clorofila (índice SPAD), observou-se aos 7 e 14 DAA que em ambos os híbridos os menores índices foram registrados para todas as misturas em tanque dos herbicidas na ausência do FEP (Tabela 2). Esses resultados demonstram potencial do FEP em auxiliar na manutenção inicial do teor de clorofila para valores próximo aos das testemunhas, quando as plantas de milho foram submetidas às associações dos herbicidas. Entretanto, apesar do FEP ter melhorado essa variável aos 7 e 14 DAA, os resultados ainda assim tiveram índices SPAD inferiores às testemunhas sem aplicação. A partir de 21 DAA, em ambos os híbridos, os teores de clorofila não mais diferiram significativamente entre si para todos os tratamentos. Resultados semelhantes foram descritos por Silva et al. (2010), os quais observaram a recuperação dos danos fitotóxicos causados por nicosulfuron em plantas de milho resultantes da aplicação de aminoácidos, utilizado na formulação comercial Kadostin ${ }^{\circledR}$.

De acordo com Hamza e Suggars (2001), os bioestimulantes e as substâncias húmicas têm demonstrado influência em processos metabólicos nas plantas, podendo aumentar o conteúdo de clorofila das células e, consequentemente, proporcionar folhas mais verdes e diminuição de problemas nas plantas, como clorose, uma vez que essas substâncias têm 
Tabela 1. Fitointoxicação (\%) nos híbridos de milho $\mathrm{P} 2530^{\circledR}$ e DKB $330 \mathrm{PRO} 2^{\circledR}$ aos 7, 14, 21 e 28 dias após a aplicação (DAA) de misturas em tanque de herbicidas associadas ou não ao fertilizante foliar Fertiactyl Pós ${ }^{\circledR}$ (FEP). Guarapuava-PR, 2016/17.

\begin{tabular}{|c|c|c|c|c|c|c|c|c|}
\hline \multirow{3}{*}{ Tratamentos } & \multicolumn{8}{|c|}{ Fitointoxicação (\%) - híbrido P2530 } \\
\hline & \multicolumn{2}{|c|}{$7 \mathrm{DAA}$} & \multicolumn{2}{|c|}{14 DAA } & \multicolumn{2}{|c|}{$21 \mathrm{DAA}$} & \multicolumn{2}{|c|}{$28 \mathrm{DAA}$} \\
\hline & sem FEP & $\begin{array}{l}\text { com } \\
\text { FEP }\end{array}$ & sem FEP & $\begin{array}{l}\text { com } \\
\text { FEP }\end{array}$ & sem FEP & com FEP & sem FEP & com FEP \\
\hline $\mathrm{ATR}^{1 /}+\mathrm{NIC}^{2 /}$ & 10,6 aA & $7,4 \mathrm{aB}$ & $8,4 \mathrm{aA}$ & $4,6 \mathrm{aB}$ & $5,2 \mathrm{aA}$ & $1,8 \mathrm{aB}$ & 0,0 & 0,0 \\
\hline $\mathrm{ATR}+\mathrm{MES}^{3 /}$ & $9,2 \mathrm{aA}$ & $6,2 \mathrm{aB}$ & $6,8 \mathrm{aA}$ & $3,8 \mathrm{aB}$ & $3,8 \mathrm{aA}$ & $1,2 \mathrm{aB}$ & 0,0 & 0,0 \\
\hline $\mathrm{ATR}+\mathrm{TEM}^{4 /}$ & $10,2 \mathrm{aA}$ & $6,8 \mathrm{aB}$ & $8,2 \mathrm{aA}$ & $4,2 \mathrm{aB}$ & $5,2 \mathrm{aA}$ & $1,2 \mathrm{aB}$ & 0,0 & 0,0 \\
\hline Teste capinada & \multicolumn{2}{|c|}{$0,0 \mathrm{~b}$} & \multicolumn{2}{|c|}{$0,0 \mathrm{~b}$} & \multicolumn{2}{|c|}{$0,0 \mathrm{~b}$} & \multicolumn{2}{|c|}{0,0} \\
\hline Teste sem capina & \multicolumn{2}{|c|}{$0,0 \mathrm{~b}$} & \multicolumn{2}{|c|}{$0,0 \mathrm{~b}$} & \multicolumn{2}{|c|}{$0,0 \mathrm{~b}$} & \multicolumn{2}{|c|}{0,0} \\
\hline Fcal herbicidas & \multicolumn{2}{|c|}{$104,681^{*}$} & \multicolumn{2}{|c|}{$68,957^{*}$} & \multicolumn{2}{|c|}{$12,600^{*}$} & \multicolumn{2}{|c|}{-} \\
\hline Fcal $_{\text {FEP }}$ & \multicolumn{2}{|c|}{$33,635^{*}$} & \multicolumn{2}{|c|}{$54,287^{*}$} & \multicolumn{2}{|c|}{$31,167^{*}$} & \multicolumn{2}{|c|}{-} \\
\hline Fcal herbicidas x FEP & \multicolumn{2}{|c|}{$3,776^{*}$} & \multicolumn{2}{|c|}{$6,379 *$} & \multicolumn{2}{|c|}{$3,873^{*}$} & \multicolumn{2}{|c|}{-} \\
\hline $\mathrm{CV}(\%)$ & \multicolumn{2}{|c|}{20,77} & \multicolumn{2}{|c|}{25,75} & \multicolumn{2}{|c|}{61,57} & \multicolumn{2}{|c|}{-} \\
\hline
\end{tabular}

Fitointoxicação (\%) - híbrido DKB 330 PRO2 ${ }^{\circledR}$

\begin{tabular}{|c|c|c|c|c|c|c|c|c|}
\hline $\mathrm{ATR}^{1 /}+\mathrm{NIC}^{2 /}$ & $11,8 \mathrm{aA}$ & $6,2 \mathrm{aB}$ & $9,2 \mathrm{aA}$ & $3,8 \mathrm{aB}$ & $5,6 \mathrm{aA}$ & $1,2 \mathrm{bB}$ & 0,0 & 0,0 \\
\hline $\mathrm{ATR}+\mathrm{MES}^{3 /}$ & $9,2 \mathrm{aA}$ & $5,6 \mathrm{aB}$ & $6,8 \mathrm{aA}$ & $3,4 \mathrm{aB}$ & $4,2 \mathrm{aA}$ & $0,6 \mathrm{bB}$ & 0,0 & 0,0 \\
\hline $\mathrm{ATR}+\mathrm{TEM}^{4 /}$ & $10,6 \mathrm{aA}$ & $6,2 \mathrm{aB}$ & $8,4 \mathrm{aA}$ & $3,8 \mathrm{aB}$ & $4,6 \mathrm{aA}$ & $1,2 \mathrm{bB}$ & 0,0 & 0,0 \\
\hline Teste capinada & \multicolumn{2}{|c|}{$0,0 \mathrm{~b}$} & \multicolumn{2}{|c|}{$0,0 \mathrm{~b}$} & \multicolumn{2}{|c|}{$0,0 \mathrm{~b}$} & \multicolumn{2}{|c|}{0,0} \\
\hline Teste sem capina & \multicolumn{2}{|c|}{$0,0 \mathrm{~b}$} & \multicolumn{2}{|c|}{$0,0 \mathrm{~b}$} & \multicolumn{2}{|c|}{$0,0 \mathrm{~b}$} & \multicolumn{2}{|c|}{0,0} \\
\hline Fcal herbicidas & \multicolumn{2}{|c|}{$124,350^{*}$} & \multicolumn{2}{|c|}{$99,559^{*}$} & \multicolumn{2}{|c|}{$17,479^{*}$} & \multicolumn{2}{|c|}{-} \\
\hline Fcal $_{\text {FEP }}$ & \multicolumn{2}{|c|}{$82,048^{*}$} & \multicolumn{2}{|c|}{$123,470 *$} & \multicolumn{2}{|c|}{$62,567^{*}$} & \multicolumn{2}{|c|}{-} \\
\hline Fcal herbicidas x FEP & \multicolumn{2}{|c|}{$10,315^{*}$} & \multicolumn{2}{|c|}{$15,577 *$} & \multicolumn{2}{|c|}{$7,311^{*}$} & \multicolumn{2}{|c|}{-} \\
\hline CV (\%) & \multicolumn{2}{|c|}{19,14} & \multicolumn{2}{|c|}{21,55} & \multicolumn{2}{|c|}{52,39} & \multicolumn{2}{|c|}{-} \\
\hline
\end{tabular}

$-{ }^{1 /} \mathrm{ATR}=$ atrazine $\left(1000 \mathrm{~g}\right.$ i.a. ha ${ }^{-1}$ Gesaprim $\left.^{\mathbb{\circledR}}\right) ;{ }^{2} \mathrm{NIC}=$ nicosulfuron $\left(45 \mathrm{~g}\right.$ i.a. ha $^{-1}$ Accent $\left.^{\mathbb{8}}\right) ;{ }^{3 /} \mathrm{MES}=$ mesotrione $\left(100,8 \mathrm{~g}\right.$ i.a. ha ${ }^{-1}$ Callisto $\left.{ }^{\circledR}\right) ;{ }^{4} \mathrm{TEM}=$ tembotrione $\left(144 \mathrm{~g}\right.$ i.a. $\mathrm{ha}^{-1}$ Soberan $\left.^{\circledR}\right){ }^{5} \mathrm{FEP}=$ Fertiactyl Pós ${ }^{\circledR}\left(400 \mathrm{~mL} \mathrm{ha}^{-1}\right)$. Em todos os tratamentos foram adicionados os adjuvantes Desadere ${ }^{\circledR}(0,1 \%)+$ AllerBiW $^{\circledR}(0,05 \%)$.

- Médias seguidas pela mesma letra minúscula nas linhas e maiúsculas nas colunas não diferem estatisticamente entre si pelo teste de Tukey $(\mathrm{p}<0,05 \%) . *=$ Significativo e $\mathrm{NS}^{\mathrm{N}}=$ não significativo. 
capacidade de absorção de nutrientes pelas raízes. Nesse contexto, o FEP é um produto à base de aminoácidos que contém bioestimulante composto por uma fração orgânica selecionada para fornecer ácidos húmicos e fúlvicos, glicina-betaína e zeatina e uma fração mineral (Santos et al., 2013b). Sua recomendação tem sido concentrada principalmente para culturas que utilizam o glyphosate no manejo das plantas daninhas, sendo uma de suas vantagens o incremento dos níveis de clorofila (Timac Agro, 2014).

Em relação à altura das plantas de milho, com exceção do ATR + NIC sem associação ao FEP, não foram constatadas diferenças significativas entre os tratamentos aplicados e a testemunha capinada para os híbridos P2530 e DBK $330 \mathrm{PRO}^{\circledR}$, na avaliação realizada no final do ciclo da cultura (Tabela 3). Para altura de inserção das espigas de milho apenas o híbrido DBK 330 PRO ${ }^{\circledR}$ com aplicação de ATR + TEM sem associação ao FEP apresentou altura inferior à testemunha capinada (Tabela 3 ). De forma geral, apesar de os dados apresentarem apenas interferência significativa entre as associações de herbicidas para a altura de plantas e de inserção das espigas, as diferenças mínimas aceitáveis foram na prática quase que imperceptíveis para os dois híbridos, o que indica baixa influência dos tratamentos herbicidas com ou sem a associação ao FEP sobre essas variáveis.

Outros resultados de pesquisas também demonstraram não ter ocorrido interferência significativa para a variável altura das plantas de milho quando submetidas à aplicação de ATR + NIC, ATR + MES e ATR + TEM (Nicolai et al., 2006; Portugal, 2013; Giovanelli et al., 2018). Entretanto, Santos et al. (2015) observaram redução linear da altura das plantas de soja M-SOY $9144^{\circledR}$ em função da aplicação de diferentes doses de FEP associado com glyphosate + lactofen. Esse resultado pode ter ocorrido por causa da potencialização dos efeitos fitotóxicos para a referida mistura de herbicidas causada pelo incremento da dose do fertilizante foliar.

Para as variáveis dos componentes da produção relacionadas ao número de fileira de grãos nas espigas (NFG) e peso de 100 grãos (P100G) não foram encontradas diferenças significativas entre os tratamentos para os dois híbridos estudados (Tabela 4). Entretanto, para o número de grãos por fileiras nas espigas (NGF), os tratamentos com ATR + NIC associados ou não ao FEP foram significativamente inferiores à testemunha capinada apenas para o híbrido DKB 330 PRO2. Algumas pesquisas indicam que esse efeito deletério pode estar associado à sensibilidade diferencial dos híbridos de milho ao nicosulfuron (Contiero, 2009; Espanhol, 2009), assim como à aplicação tardia dos herbicidas que podem afetar a diferenciação floral da panícula e, consequentemente, componentes da produção tais como o NFG e a quantidade de grãos por espiga (Andrade et al., 1996).

Spader e Vidal (2001), López-Ovejero et al. (2003) e Cavalieri et al. (2012) mencionaram que o número de grãos nas espigas e em suas fileiras foram reduzidos em plantas de milho com nicosulfuron aplicado nos estádios fenológicos V7 a V9, diferentemente de V3 a V6. Já Portugal (2013) relatou não ter ocorrido diferença significativa para a altura da espiga, número de grãos por espiga e NFG, para aplicação das associações dos herbicidas ATR + NIC, ATR + MES e ATR + TEM. A convivência das plantas daninhas durante todo o ciclo da cultura do milho reduziu significativamente o NGF (16,3\%) apenas para o híbrido DKB 330 PRO2 (Tabela 4).

Em relação à produtividade de grãos (PROD), todos os tratamentos diferiram apenas em relação à testemunha sem capina dos híbridos P2530 e DKB330 PRO2 ${ }^{\circledR}$, os quais tiveram redução em média 
Tabela 2. Teor de clorofila (índice SPAD) nos híbridos de milho P2530 ${ }^{\circledR}$ e DKB 330 PRO2 ${ }^{\circledR}$ aos 7, 14, 21 e 28 dias após a aplicação (DAA) de misturas em tanque de herbicidas associadas ou não ao fertilizante foliar Fertiactyl Pós ${ }^{\circledR}$ (FEP). Guarapuava-PR, 2016/17.

\begin{tabular}{|c|c|c|c|c|c|c|c|c|}
\hline \multirow{4}{*}{ Tratamentos } & \multicolumn{8}{|c|}{ Teor de clorofila (índice SPAD) - híbrido P2530 ${ }^{\circledR}$} \\
\hline & \multicolumn{2}{|c|}{$7 \mathrm{DAA}$} & \multicolumn{2}{|c|}{$14 \mathrm{DAA}$} & \multicolumn{2}{|c|}{$21 \mathrm{DAA}$} & \multicolumn{2}{|c|}{$28 \mathrm{DAA}$} \\
\hline & \multirow{2}{*}{ sem FEP } & \multirow{2}{*}{$\begin{array}{l}\text { com } \\
\text { FEP }\end{array}$} & \multirow{2}{*}{ sem FEP } & \multirow{2}{*}{$\begin{array}{l}\text { com } \\
\text { FEP }\end{array}$} & \multirow{2}{*}{ sem FEP } & \multirow{2}{*}{ com FEP } & \multirow{2}{*}{ sem FEP } & \multirow{2}{*}{ com FEP } \\
\hline & & & & & & & & \\
\hline $\mathrm{ATR}^{1 /}+\mathrm{NIC}^{2 /}$ & $27,3 \mathrm{bB}$ & $29,0 \mathrm{bA}$ & $30,4 \mathrm{bB}$ & $32,3 \mathrm{bA}$ & 35,7 & 36,2 & 39,3 & 39,0 \\
\hline $\mathrm{ATR}+\mathrm{MES}^{3 /}$ & $27,5 \mathrm{bB}$ & $29,4 \mathrm{bA}$ & $30,9 \mathrm{bB}$ & $32,1 \mathrm{bA}$ & 35,8 & 36,2 & 38,7 & 39,3 \\
\hline $\mathrm{ATR}+\mathrm{TEM}^{4 /}$ & $27,7 \mathrm{bB}$ & $29,1 \mathrm{bA}$ & $30,2 \mathrm{bB}$ & $32,8 \mathrm{bA}$ & 35,9 & 36,0 & 39,4 & 38,9 \\
\hline Teste capinada & \multicolumn{2}{|c|}{$33,5 \mathrm{a}$} & \multicolumn{2}{|c|}{$34,2 \mathrm{a}$} & \multicolumn{2}{|c|}{36,6} & \multicolumn{2}{|c|}{39,7} \\
\hline Teste sem capina & \multicolumn{2}{|c|}{$32,7 \mathrm{a}$} & \multicolumn{2}{|c|}{$34,4 \mathrm{a}$} & \multicolumn{2}{|c|}{36,0} & \multicolumn{2}{|c|}{39,3} \\
\hline Fcal herbicidas & \multicolumn{2}{|c|}{$213,841 *$} & \multicolumn{2}{|c|}{$44,146^{*}$} & \multicolumn{2}{|c|}{$0,981^{\mathrm{NS}}$} & \multicolumn{2}{|c|}{$1,028^{\mathrm{NS}}$} \\
\hline Fcal $_{\text {FEP }}$ & \multicolumn{2}{|c|}{$41,719^{*}$} & \multicolumn{2}{|c|}{$45,731 *$} & \multicolumn{2}{|c|}{$0,329^{\mathrm{NS}}$} & \multicolumn{2}{|c|}{$0,497^{\mathrm{NS}}$} \\
\hline Fcal herbicidas x FEP & \multicolumn{2}{|c|}{$14,406^{*}$} & \multicolumn{2}{|c|}{$5,795^{*}$} & \multicolumn{2}{|c|}{$2.027^{\mathrm{NS}}$} & 1,7 & $4^{\mathrm{NS}}$ \\
\hline CV $(\%)$ & & & & & & 61 & & 72 \\
\hline & & Teor $\mathrm{C}$ & clorofila & 1dice SPA & ) - híbric & DKB 330 & $\mathrm{PRO}^{(B)}$ & \\
\hline $\mathrm{ATR}^{1 /}+\mathrm{NIC}^{2 /}$ & $27,9 \mathrm{bB}$ & $29,5 \mathrm{bA}$ & $30,6 \mathrm{bB}$ & $31,9 \mathrm{bA}$ & 35,2 & 35,9 & 38,8 & 39,6 \\
\hline $\mathrm{ATR}+\mathrm{MES}^{3 /}$ & $27,8 \mathrm{bB}$ & $28,9 \mathrm{bA}$ & $30,1 \mathrm{bB}$ & $32,5 \mathrm{bA}$ & 35,9 & 35,9 & 38,9 & 39,2 \\
\hline $\mathrm{ATR}+\mathrm{TEM}^{4 /}$ & $27,4 \mathrm{bB}$ & $29,2 \mathrm{bA}$ & $30,3 \mathrm{bB}$ & $32,3 \mathrm{bA}$ & 35,2 & 35,9 & 38,5 & 39,2 \\
\hline Teste capinada & & & & & & 6,4 & & ,4 \\
\hline Teste sem capina & & & & & & 5 & & 0 \\
\hline Fcal herbicidas & 94, & $57^{*}$ & 38 & $40^{*}$ & & $8^{\mathrm{NS}}$ & 0,5 & $2^{\mathrm{NS}}$ \\
\hline Fcal FEP & 12 , & $16^{*}$ & 28 , & $55^{*}$ & & $45^{\mathrm{NS}}$ & 3,5 & $2^{\mathrm{NS}}$ \\
\hline Fcal herbicidas x FEP & & & 6,4 & & & $17^{\mathrm{NS}}$ & 2,3 & $0^{\mathrm{NS}}$ \\
\hline $\mathrm{CV}(\%)$ & & & & & & 17 & & 47 \\
\hline
\end{tabular}

$-{ }^{1 /} \mathrm{ATR}=$ atrazine $\left(1000 \mathrm{~g}\right.$ i.a. ha $^{-1}$ Gesaprim $\left.{ }^{\circledR}\right) ;{ }^{2} \mathrm{NIC}=$ nicosulfuron $\left(45 \mathrm{~g}\right.$ i.a. ha ${ }^{-1}$ Accent $\left.^{\circledR}\right) ;{ }^{3 / \mathrm{MES}}=$ mesotrione $\left(100,8\right.$ g i.a. ha ${ }^{-1}$ Callisto $\left.^{\circledR}\right) ;{ }^{4 / T E M}=$ tembotrione $\left(144\right.$ g i.a. ha ${ }^{-1}$ Soberan $\left.^{\circledR}\right){ }^{5 /} \mathrm{FEP}=$ Fertiactyl Pós ${ }^{\circledR}(400 \mathrm{~mL}$ ha $\left.^{-1}\right)$. Em todos os tratamentos foram adicionados os adjuvantes Desadere ${ }^{\circledR}(0,1 \%)+$ AllerBiW $^{\circledR}(0,05 \%)$.

- Médias seguidas pela mesma letra minúscula nas linhas e maiúsculas nas colunas não diferem estatisticamente entre si pelo teste de Tukey $(\mathrm{p}<0,05 \%) . *$ Significativo $\mathrm{e}^{\mathrm{NS}}=$ não significativo. 
Tabela 3. Altura das plantas (AP) e de inserção das espigas (AE) no final do ciclo nos híbridos de milho P2530 ${ }^{\circledR}$ e DKB 330 PRO $2^{\circledR}$ submetidos a misturas em tanque de herbicidas associadas ou não ao fertilizante foliar Fertiactyl Pós ${ }^{\circledR}$ (FEP). Guarapuava - PR, 2016/17.

\begin{tabular}{|c|c|c|c|c|c|c|c|c|}
\hline \multirow{3}{*}{ Tratamentos } & \multicolumn{4}{|c|}{ híbrido $\mathrm{P} 2530^{\circledR}$} & \multicolumn{4}{|c|}{ híbrido DKB 330 PRO2 ${ }^{\circledR}$} \\
\hline & \multicolumn{2}{|c|}{$\mathrm{AP}(\mathrm{m})$} & \multicolumn{2}{|c|}{$\mathrm{AE}(\mathrm{m})$} & \multicolumn{2}{|c|}{$\mathrm{AP}$} & \multicolumn{2}{|c|}{$\mathrm{AE}$} \\
\hline & sem FEP & $\begin{array}{l}\text { com } \\
\text { FEP }\end{array}$ & sem FEP & $\begin{array}{l}\text { com } \\
\text { FEP }\end{array}$ & sem FEP & com FEP & sem FEP & com FEP \\
\hline $\mathrm{ATR}^{1 /}+\mathrm{NIC}^{2 /}$ & $2,43 \quad b$ & $2,45 \mathrm{a}$ & $1,29 \mathrm{a}$ & $1,29 \mathrm{a}$ & $2,35 \mathrm{~b}$ & $2,37 \mathrm{a}$ & $1,27 \mathrm{ab}$ & $1,27 \mathrm{ab}$ \\
\hline $\mathrm{ATR}+\mathrm{MES}^{3 /}$ & $2,44 \mathrm{ab}$ & $2,44 \mathrm{ab}$ & $1,29 \mathrm{a}$ & $1,29 \mathrm{a}$ & $2,36 \mathrm{ab}$ & $2,37 \mathrm{a}$ & $1,27 \mathrm{ab}$ & $1,27 \mathrm{ab}$ \\
\hline $\mathrm{ATR}+\mathrm{TEM}^{4 /}$ & $2,45 \mathrm{ab}$ & $2,45 \mathrm{a}$ & $1,29 \mathrm{a}$ & $1,30 \mathrm{a}$ & $2,36 \mathrm{ab}$ & $2,36 \mathrm{ab}$ & $1,26 \mathrm{~b}$ & $1,27 \mathrm{ab}$ \\
\hline Teste capinada & \multicolumn{2}{|c|}{$2,46 \mathrm{a}$} & \multicolumn{2}{|c|}{$1,29 \mathrm{a}$} & \multicolumn{2}{|c|}{$2,38 \mathrm{a}$} & \multicolumn{2}{|c|}{$1,29 \mathrm{a}$} \\
\hline Teste sem capina & \multicolumn{2}{|c|}{$2,44 \mathrm{ab}$} & \multicolumn{2}{|c|}{$1,27 \mathrm{~b}$} & \multicolumn{2}{|c|}{$2,36 \mathrm{ab}$} & \multicolumn{2}{|c|}{$1,26 b$} \\
\hline Fcal herbicidas & \multicolumn{2}{|c|}{$3,637^{*}$} & \multicolumn{2}{|c|}{$8,808^{*}$} & \multicolumn{2}{|c|}{$4,541^{*}$} & \multicolumn{2}{|c|}{$6,835^{*}$} \\
\hline Fcal FEP & \multicolumn{2}{|c|}{$0,769^{\mathrm{NS}}$} & \multicolumn{2}{|c|}{$0,649^{\mathrm{NS}}$} & \multicolumn{2}{|c|}{$3,784^{\mathrm{NS}}$} & \multicolumn{2}{|c|}{$0,069^{\mathrm{NS}}$} \\
\hline Fcal herbicidas x FEP & \multicolumn{2}{|c|}{$1,143^{\mathrm{NS}}$} & \multicolumn{2}{|c|}{$0,619^{\mathrm{NS}}$} & \multicolumn{2}{|c|}{$1,120^{\mathrm{NS}}$} & \multicolumn{2}{|c|}{$0,122^{\mathrm{NS}}$} \\
\hline $\mathrm{CV}(\%)$ & \multicolumn{2}{|c|}{0,59} & \multicolumn{2}{|c|}{0,87} & \multicolumn{2}{|c|}{0,72} & \multicolumn{2}{|c|}{0,93} \\
\hline
\end{tabular}

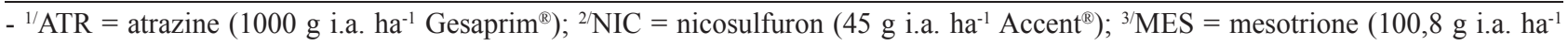
Callisto $\left.{ }^{\circledR}\right) ;{ }^{4}$ TEM $=$ tembotrione $\left(144\right.$ g i.a. ha $^{-1}$ Soberan $\left.{ }^{\circledR}\right){ }^{5 / F E P}=$ Fertiactyl Pós ${ }^{\circledR}\left(400 \mathrm{~mL} \mathrm{ha}^{-1}\right)$. Em todos os tratamentos foram adicionados os adjuvantes Desadere ${ }^{\circledR}(0,1 \%)+$ AllerBiW $^{\circledR}(0,05 \%)$.

- Médias seguidas pela mesma letra minúscula nas linhas e maiúsculas nas colunas não diferem estatisticamente entre si pelo teste de Tukey $(\mathrm{p}<0,05 \%){ }^{*}=$ Significativo $\mathrm{e}^{\mathrm{NS}}=$ não significativo.

na ordem de $35 \%$ e $38 \%$, respectivamente (Tabela 4). Esse resultado evidencia que todas as associações dos herbicidas foram seletivas, e que a adição de FEP em mistura em tanque com herbicidas não proporcionou incremento ou redução de PROD. Esse fato pode ser explicado pelos níveis de intoxicação das associações dos herbicidas terem sido baixos em relação aos híbridos de milho estudados, e também por não ter ocorrido nenhum outro tipo de estresse abiótico, tais como estresse hídrico, que poderia talvez influenciar negativamente a PROD dos tratamentos em relação à testemunha somente capinada.

Resultados contrastantes foram observados em trabalhos com a cultura da soja RR, desenvolvi- dos por Santos et al. (2015) e Dranca et al. (2018), observaram que a aplicação da mistura em tanque de glyphosate + lactofen + FEP incrementou a produtividade de grãos, em condições em que a cultura foi submetida a 27 dias de estresse hídrico e onde não houve estresse hídrico, respectivamente. Em ambas as condições, o fertilizante FEP, por possuir base de substâncias húmicas e aminoácidos, pode ter causado maior desenvolvimento radicular e, consequentemente, maior absorção de água e nutrientes pela cultura.

Segundo os critérios estabelecidos pela Sociedade Brasileira da Ciência das Plantas Daninhas (Velini et al., 1995), para as plantas daninhas preva- 
Tabela 4. Componentes da produção número de fileiras de grãos (NFG), número de grãos por fileiras (NGF), peso de 100 grãos (P100G) e produtividade (PROD) dos híbridos de milho P2530 ${ }^{\circledR}$ e DKB $330 \mathrm{PRO}^{\circledR}$ submetidos à aplicação de misturas em tanque de herbicidas, associadas ou não ao fertilizante foliar Fertiactyl Pós ${ }^{\circledR}$ (FEP). Guarapuava-PR, 2016/17.

\begin{tabular}{|c|c|c|c|c|c|c|c|c|}
\hline \multirow{4}{*}{ Tratamentos } & \multicolumn{8}{|c|}{ híbrido P2530 ${ }^{(R)}$} \\
\hline & \multicolumn{2}{|c|}{ NFG } & \multicolumn{2}{|c|}{ NGF } & \multicolumn{2}{|c|}{ P100G } & \multicolumn{2}{|c|}{ PROD } \\
\hline & \multirow{2}{*}{ sem FEP } & com & \multirow{2}{*}{ sem FEP } & com & sem & com & sem & com \\
\hline & & FEP & & FEP & FEP & FEP & FEP & FEP \\
\hline $\mathrm{ATR}^{1 /}+\mathrm{NIC}^{2 /}$ & 14,0 & 14,7 & $40,0 \mathrm{ab}$ & $40,5 \mathrm{ab}$ & 44,5 & 44,2 & $13101 \mathrm{ab}$ & $13224 \mathrm{a}$ \\
\hline $\mathrm{ATR}+\mathrm{MES}^{3 /}$ & 14,6 & 14,9 & $41,0 \mathrm{a}$ & $40,9 \mathrm{a}$ & 44,3 & 44,2 & $12117 \mathrm{ab}$ & $12861 \mathrm{ab}$ \\
\hline $\mathrm{ATR}+\mathrm{TEM}^{4 /}$ & 14,5 & 14,8 & $40,8 \mathrm{a}$ & $40,9 \mathrm{a}$ & 44,0 & 44,3 & $13032 \mathrm{ab}$ & $13260 \mathrm{a}$ \\
\hline Teste capinada & \multicolumn{2}{|c|}{15,0} & \multicolumn{2}{|c|}{$39,6 \mathrm{ab}$} & \multicolumn{2}{|c|}{44,3} & \multicolumn{2}{|c|}{$14160 \mathrm{a}$} \\
\hline Teste sem capina & \multicolumn{2}{|c|}{14,4} & \multicolumn{2}{|c|}{$38,4 \quad b$} & \multicolumn{2}{|c|}{43,9} & \multicolumn{2}{|c|}{$9217 \mathrm{~b}$} \\
\hline Fcal herbicidas & \multicolumn{2}{|c|}{$2.839^{\mathrm{NS}}$} & \multicolumn{2}{|c|}{$7,318^{*}$} & \multicolumn{2}{|c|}{$0,589^{\mathrm{NS}}$} & \multicolumn{2}{|c|}{$7,595^{*}$} \\
\hline Fcal FEP $_{\text {FE }}$ & \multicolumn{2}{|c|}{$3.391^{\mathrm{NS}}$} & \multicolumn{2}{|c|}{$0,080^{\mathrm{NS}}$} & \multicolumn{2}{|c|}{$0,036^{\mathrm{NS}}$} & \multicolumn{2}{|c|}{$0,127^{\mathrm{NS}}$} \\
\hline Fcal $_{\text {herbicidas x FEP }}$ & \multicolumn{2}{|c|}{$0,833^{\mathrm{NS}}$} & \multicolumn{2}{|c|}{$0,099^{\mathrm{NS}}$} & \multicolumn{2}{|c|}{$0,322^{\mathrm{NS}}$} & \multicolumn{2}{|c|}{$0,050^{\mathrm{NS}}$} \\
\hline $\mathrm{CV}(\%)$ & \multicolumn{2}{|c|}{3,41} & \multicolumn{2}{|c|}{3,01} & \multicolumn{2}{|c|}{1,51} & \multicolumn{2}{|c|}{17,49} \\
\hline
\end{tabular}

\begin{tabular}{|c|c|c|c|c|c|c|c|c|}
\hline & \multicolumn{8}{|c|}{ híbrido DKB 330 PRO2 ${ }^{(B)}$} \\
\hline $\mathrm{ATR}^{1 /}+\mathrm{NIC}^{2 /}$ & 15,4 & 15,8 & $34,8 \quad b$ & $35,6 \mathrm{bc}$ & 44,6 & 43,9 & $10820 \mathrm{ab}$ & $10364 \mathrm{ab}$ \\
\hline $\mathrm{ATR}+\mathrm{MES}^{3 /}$ & 15,3 & 15,9 & $37,5 \mathrm{a}$ & $37,1 \mathrm{a}$ & 44,6 & 44,2 & $11611 \mathrm{a}$ & $11172 \mathrm{a}$ \\
\hline $\mathrm{ATR}+\mathrm{TEM}^{4 /}$ & 15,4 & 15,5 & $36,3 \mathrm{ab}$ & $37,0 \mathrm{a}$ & 44,4 & 44,6 & $11315 \mathrm{a}$ & $11155 \mathrm{a}$ \\
\hline Teste capinada & \multicolumn{2}{|c|}{15,5} & \multicolumn{2}{|c|}{$37,4 \mathrm{a}$} & \multicolumn{2}{|c|}{44,8} & \multicolumn{2}{|c|}{$11994 \mathrm{a}$} \\
\hline Teste sem capina & \multicolumn{2}{|c|}{15,4} & \multicolumn{2}{|c|}{$31,3 \mathrm{c}$} & \multicolumn{2}{|c|}{44,2} & \multicolumn{2}{|c|}{$8761 \mathrm{~b}$} \\
\hline Fcal herbicidas & \multicolumn{2}{|c|}{$0,123^{\mathrm{NS}}$} & \multicolumn{2}{|c|}{$33,060^{*}$} & \multicolumn{2}{|c|}{$1,016^{\mathrm{NS}}$} & \multicolumn{2}{|c|}{$10,417^{*}$} \\
\hline Fcal $_{\text {FEP }}$ & \multicolumn{2}{|c|}{$0,934^{\mathrm{NS}}$} & \multicolumn{2}{|c|}{$0,395^{\mathrm{NS}}$} & \multicolumn{2}{|c|}{$0,530^{\mathrm{NS}}$} & \multicolumn{2}{|c|}{$0,376^{\mathrm{NS}}$} \\
\hline Fcal herbicidas x FEP & \multicolumn{2}{|c|}{$0,278^{\mathrm{NS}}$} & \multicolumn{2}{|c|}{$0,346^{\mathrm{NS}}$} & \multicolumn{2}{|c|}{$0,544^{\mathrm{NS}}$} & \multicolumn{2}{|c|}{$0,086^{\mathrm{NS}}$} \\
\hline CV $(\%)$ & \multicolumn{2}{|c|}{5,19} & \multicolumn{2}{|c|}{3,96} & \multicolumn{2}{|c|}{1,75} & \multicolumn{2}{|c|}{11,27} \\
\hline
\end{tabular}

- ${ }^{1 / A T R}=$ atrazine $\left(1000 \mathrm{~g}\right.$ i.a. ha $^{-1}$ Gesaprim $\left.^{\circledR}\right) ;{ }^{2} \mathrm{NIC}=$ nicosulfuron $\left(45 \mathrm{~g}\right.$ i.a. ha ${ }^{-1}$ Accent $\left.^{\circledR}\right) ;{ }^{3 / M E S}=$ mesotrione $\left(100,8 \mathrm{~g}\right.$ i.a. ha ${ }^{-1}$ Callisto $\left.^{\circledR}\right) ;{ }^{4 /} \mathrm{TEM}=$ tembotrione $\left(144\right.$ g i.a. ha ${ }^{-1}$ Soberan $\left.^{\circledR}\right){ }^{5 /} \mathrm{FEP}=$ Fertiactyl Pós ${ }^{\circledR}\left(400 \mathrm{~mL} \mathrm{ha}^{-1}\right)$. Em todos os tratamentos foram adicionados os adjuvantes Desadere ${ }^{\circledR}(0,1 \%)+$ AllerBiW $^{\circledR}(0,05 \%)$.

- Médias seguidas pela mesma letra minúscula nas linhas e maiúsculas nas colunas não diferem estatisticamente entre si pelo teste de Tukey $(\mathrm{p}<0,05 \%) . *$ Significativo $\mathrm{e}^{\mathrm{NS}}=$ não significativo.

lecentes, observaram-se aos 7 DAA níveis médios de controle eficiente $(91,6 \%$ e $89,2 \%)$ de erva-quente $(S$. latifolia) e satisfatório $(86,2 \%$ e $83,2 \%)$ de guanxuma (S. rhombifolia) com as associações de ATR + MES e ATR + TEM, quando aplicadas isoladas ou asso- ciadas com FEP, respectivamente (Tabela 5). Esses tratamentos também apresentaram controle significativamente superior às associações de ATR + NIC e $\mathrm{ATR}+\mathrm{NIC}+\mathrm{FEP}$ aos 7 DAA, as quais atingiram eficiência insatisfatória $(<80 \%)$. Herbicidas inibi- 
dores da síntese de carotenoides atuam por meio do dos com atrazine (inibidor de FS II) apresentam sinerbloqueio da atividade da enzima HPPD (4-hidroxife- gismo no controle de plantas daninhas, havendo até a nil- piruvato-dioxigenase) nos cloroplastos, tais como possibilidade de utilizar menores doses (Bachiega \& mesotrione e tembotrione, os quais quando combina- Soares, 2002).

Tabela 5. Controle de erva-quente ( $S$. latifolia) e guanxuma $(S$. rhombifolia) aos 7, 14, 21 e 28 dias após a aplicação (DAA) de misturas em tanque de herbicidas associadas ou não a fertilizante foliar Fertiactyl Pós ${ }^{\circledR}$ (FEP) na cultura do milho. Guarapuava-PR, 2016/17.

\begin{tabular}{|c|c|c|c|c|c|c|c|c|}
\hline \multirow{4}{*}{ Tratamentos } & \multicolumn{8}{|c|}{ Spermacoce latifolia ( $\%$ controle) } \\
\hline & \multicolumn{2}{|c|}{$7 \mathrm{DAA}$} & \multicolumn{2}{|c|}{$14 \mathrm{DAA}$} & \multicolumn{2}{|c|}{$21 \mathrm{DAA}$} & \multicolumn{2}{|c|}{$28 \mathrm{DAA}$} \\
\hline & \multirow{2}{*}{ sem FEP } & \multirow{2}{*}{$\begin{array}{l}\text { com } \\
\text { FEP }\end{array}$} & \multirow{2}{*}{ sem FEP } & \multirow{2}{*}{$\begin{array}{l}\text { com } \\
\text { FEP }\end{array}$} & \multirow{2}{*}{ sem FEP } & \multirow{2}{*}{$\begin{array}{l}\text { com } \\
\text { FEP }\end{array}$} & \multirow{2}{*}{$\begin{array}{l}\text { sem } \\
\text { FEP }\end{array}$} & \multirow{2}{*}{$\begin{array}{l}\text { com } \\
\text { FEP }\end{array}$} \\
\hline & & & & & & & & \\
\hline $\mathrm{ATR}^{1 /}+\mathrm{NIC}^{2 /}$ & $72,8 \mathrm{dA}$ & $69,8 \mathrm{~dB}$ & $79,2 \mathrm{dA}$ & $76,0 \mathrm{~dB}$ & $86,2 \mathrm{cA}$ & $83,6 \mathrm{cB}$ & 100,0 & 100,0 \\
\hline $\mathrm{ATR}+\mathrm{MES}^{3 /}$ & $91,6 \mathrm{bA}$ & $89,2 \mathrm{bB}$ & $95,2 \mathrm{bA}$ & $93,8 \mathrm{bA}$ & $97,8 \mathrm{aA}$ & $96,2 \mathrm{abA}$ & 100,0 & 100,0 \\
\hline $\mathrm{ATR}+\mathrm{TEM}^{4 /}$ & $86,2 \mathrm{cA}$ & $83,2 \mathrm{cB}$ & $90,0 \mathrm{cA}$ & $88,4 \mathrm{cA}$ & $94,2 \mathrm{bA}$ & $92,8 \mathrm{bA}$ & 100,0 & 100,0 \\
\hline Teste capinada & \multicolumn{2}{|c|}{$100,0 \mathrm{a}$} & \multicolumn{2}{|c|}{$100,0 \mathrm{a}$} & \multicolumn{2}{|c|}{$100,0 \mathrm{a}$} & \multicolumn{2}{|c|}{100,0} \\
\hline Teste sem capina & \multicolumn{2}{|c|}{$0,0 \mathrm{e}$} & \multicolumn{2}{|c|}{$0,0 \mathrm{e}$} & \multicolumn{2}{|c|}{$0,0 \mathrm{~d}$} & \multicolumn{2}{|c|}{0,0} \\
\hline Fcal herbicidas & \multicolumn{2}{|c|}{$4976,11^{*}$} & \multicolumn{2}{|c|}{$5781,07^{*}$} & \multicolumn{2}{|c|}{$10739,86^{*}$} & \multicolumn{2}{|c|}{-} \\
\hline Fcal $_{\text {FEP }}$ & \multicolumn{2}{|c|}{$10,919 *$} & \multicolumn{2}{|c|}{$6,535^{*}$} & \multicolumn{2}{|c|}{$9,389^{*}$} & \multicolumn{2}{|c|}{-} \\
\hline Fcal herbicidas x FEP & \multicolumn{2}{|c|}{$1,866^{\mathrm{NS}}$} & \multicolumn{2}{|c|}{$1,503^{\mathrm{NS}}$} & \multicolumn{2}{|c|}{$1,874^{\mathrm{NS}}$} & \multicolumn{2}{|c|}{-} \\
\hline $\mathrm{CV}(\%)$ & \multicolumn{2}{|c|}{2,59} & \multicolumn{2}{|c|}{2,37} & \multicolumn{2}{|c|}{1,72} & \multicolumn{2}{|c|}{-} \\
\hline
\end{tabular}

Sida rhombifolia $(\%$ controle $)$

\begin{tabular}{|c|c|c|c|c|c|c|c|c|}
\hline $\mathrm{ATR}^{1 /}+\mathrm{NIC}^{2 /}$ & $66,8 \mathrm{c}$ & $65,8 \mathrm{c}$ & $74,2 \mathrm{c}$ & $73,2 \mathrm{c}$ & $81,6 \mathrm{c}$ & $80,2 \mathrm{c}$ & 100,0 & 100,0 \\
\hline $\mathrm{ATR}+\mathrm{MES}^{3 /}$ & $88,8 \mathrm{~b}$ & $87,8 \mathrm{~b}$ & $93,6 \mathrm{~b}$ & $92,4 \mathrm{~b}$ & $97,0 \mathrm{~b}$ & $95,2 \mathrm{~b}$ & 100,0 & 100,0 \\
\hline $\mathrm{ATR}+\mathrm{TEM}^{4 /}$ & $84,2 \mathrm{~b}$ & $84,8 \mathrm{~b}$ & $90,8 \mathrm{~b}$ & $88,2 \mathrm{~b}$ & 94,6 b & $92,8 \mathrm{~b}$ & 100,0 & 100,0 \\
\hline Teste capinada & \multicolumn{2}{|c|}{$100,0 \mathrm{a}$} & \multicolumn{2}{|c|}{$100,0 \mathrm{a}$} & \multicolumn{2}{|c|}{$100,0 \mathrm{a}$} & \multicolumn{2}{|c|}{100,0} \\
\hline Teste sem capina & \multicolumn{2}{|c|}{$0,0 \mathrm{~d}$} & \multicolumn{2}{|c|}{$0,0 \mathrm{~d}$} & \multicolumn{2}{|c|}{$0,0 \mathrm{~d}$} & \multicolumn{2}{|c|}{0,0} \\
\hline Fcal herbicidas & \multicolumn{2}{|c|}{$3912,68 *$} & \multicolumn{2}{|c|}{$4646,16^{*}$} & \multicolumn{2}{|c|}{$9326,90 *$} & \multicolumn{2}{|c|}{-} \\
\hline Fcal $_{\text {FEP }}$ & \multicolumn{2}{|c|}{$0,242^{\mathrm{NS}}$} & \multicolumn{2}{|c|}{$3,188^{\mathrm{NS}}$} & \multicolumn{2}{|c|}{$6,591^{\mathrm{NS}}$} & \multicolumn{2}{|c|}{-} \\
\hline Fcal herbicidas x FEP & \multicolumn{2}{|c|}{$0,304^{\mathrm{NS}}$} & \multicolumn{2}{|c|}{$0,794^{\mathrm{NS}}$} & \multicolumn{2}{|c|}{$1,134^{\mathrm{NS}}$} & \multicolumn{2}{|c|}{-} \\
\hline CV (\%) & \multicolumn{2}{|c|}{2,97} & \multicolumn{2}{|c|}{2,67} & \multicolumn{2}{|c|}{1,86} & \multicolumn{2}{|c|}{-} \\
\hline
\end{tabular}

$-{ }^{1 /} \mathrm{ATR}=$ atrazine $\left(1000 \mathrm{~g}\right.$ i.a. ha ${ }^{-1}$ Gesaprim $\left.^{\circledR}\right) ;{ }^{2} \mathrm{NIC}=$ nicosulfuron $\left(45 \mathrm{~g}\right.$ i.a. ha $^{-1}$ Accent $\left.{ }^{\circledR}\right) ;{ }^{3} \mathrm{MES}=$ mesotrione $\left(100,8 \mathrm{~g}\right.$ i.a. ha ${ }^{-1}$ Callisto $\left.{ }^{\circledR}\right) ;{ }^{4}$ TEM $=$ tembotrione $\left(144\right.$ g i.a. ha $^{-1}$ Soberan $\left.{ }^{\circledR}\right){ }^{5} \mathrm{FEP}=$ Fertiactyl Pós ${ }^{\circledR}\left(400 \mathrm{~mL} \mathrm{ha}^{-1}\right)$. Em todos os tratamentos foram adicionados os adjuvantes Desadere ${ }^{\circledR}(0,1 \%)+\operatorname{AllerBiW}^{\circledR}(0,05 \%)$.

- Médias seguidas pela mesma letra minúscula nas linhas e maiúsculas nas colunas não diferem estatisticamente entre si pelo teste de Tukey $(\mathrm{p}<0,05 \%) . *$ Significativo e ${ }^{\mathrm{NS}}=$ não significativo. 
Aos 14 e 21 DAA, as associações de ATR + MES e ATR + TEM, aplicadas isoladas ou associadas com FEP, registraram controle das plantas daninhas com sendo altamente eficiente (>90\%) e significativamente superior a ATR + NIC e ATR + NIC + FEP, os quais atingiram no máximo apenas controle satisfatório (> 80\%) no referido período (Tabela 5). Outros resultados de pesquisa também demonstraram que a velocidade de ação no controle de plantas daninhas de associações de ATR + MES e ATR + TEM é superior a ATR + NIC, e que pode ainda ser influenciada pelo estádio de desenvolvimento da infestação (Parker et al. 2006; Rizzardi et al., 2008; Oliveira Neto et al., 2011).

É importante ressaltar que a mistura em tanque de FEP resultou em controle de erva-quente ( $S$. latifolia) significativamente inferior somente aos 7 DAA quando associada aos herbicidas ATR + MES e ATR + TEM, assim como dos 7 aos 21 DAA com ATR + NIC. Entretanto, aos 28 DAA todos os tratamentos atingiram $100 \%$ de controle de erva-quente e guanxuma. De forma geral, os destaques no manejo das plantas daninhas na cultura do milho foram a maior velocidade de ação no controle inicial de erva-quente com associação dos herbicidas ATR + MES, assim como a não interferência do FEP na eficiência final dos tratamentos estudados.

\section{Conclusão}

A associação do fertilizante foliar Fertiactyl Pós ${ }^{\circledR}$ em misturas em tanque com os herbicidas atrazine + nicossulfuron, atrazine + mesotrione e atrazine + tembotrione não interferiu na deposição da pulverização em milho e erva-quente, assim como na eficiência de controle de plantas daninhas e na produtividade de grãos.
Fertiactyl Pós ${ }^{\circledR}$ pode ser utilizado em mistura em tanque com herbicidas para redução da fitointoxicação e melhoria dos níveis de clorofila na cultura do milho.

\section{Referências}

ALVES, B. M.; CARGNELUTTI FILHO, A.; TOEBE, C. B. M.; SILVA, P. L. Divergência genética de milho transgênico em relação à produtividade de grãos e da qualidade nutricional. Ciência Rural, v. 45, n. 5, p. 884891, 2015. DOI: 10.1590/0103-8478cr20140471.

ANDRADE, F.; CIRILO, A.; UHARTS, S.; OTEGUI, M. Ecofisiologia de cultivo de maíz. Balcaral: La Barresa, 1996. $392 \mathrm{p}$.

BACHIEGA, A. L.; SOARES, J. E. Callisto (mesotrione): novo herbicida para o controle de plantas daninhas em pós-emergência, na cultura do milho. In: CONGRESSO BRASILEIRO DA CIÊNCIA DAS PLANTAS DANINHAS, 23., 2002, Londrina. Resumos... Londrina: SBCPD; Pelotas: Embrapa Clima Temperado, 2002.p. 655.

CALVO, P.; NELSON, L.; KLOEPPER, J. W. Agricultural uses of plant biostimulants. Plant Soil, v. 383, n. 1/2, p. 3-41, 2014. DOI: 10.1007/s11104-014-2131-8.

CARVAlHO, F. T.; MORETTI, T. B.; SOUZA, P. A. Eficácia e seletividade de associações de herbicidas utilizados em pós-emergência na cultura do milho. Revista Brasileira de Herbicidas, v. 9, n. 2, p. 35-41, 2010. DOI: $10.7824 /$ rbh.v9i2.79.

CAVALIERI, S. D.; SILVA, F. M. L.; VELINI, E. D.; SÃO JOSÉ, A. R.; ULLOA, S. M.; DATTA, A.; CAVALIERI, J. D.; KNEZEVIC, S. Z. Seletividade do nicosulfuron em três estádios fenológicos de milho-pipoca. Planta Daninha, v. 30, n. 2, p. 377-386, 2012.

DOI: $10.1590 / \mathrm{S} 0100-83582012000200017$.

CONSTANTIN, J.; OLIBEIRA JÚNIOR, R. S.; GHENO, E. A.; BIFFE, D. F.; BRAZ, G. B. P.; WEBER, F.; TAKANO, H. K. Prevention of yield losses caused 
by glyphosate in soybeans with biostimulant. African Journal of Agricultural Research, v. 11, n. 18, p. 1601$1607,2016$.

DOI: $10.5897 / A J A R 2016.10809$.

CONTIERO, R. L. Seletividade dos herbicidas nicosulfuron e foramsulfuron + iodosulfuron methyl sodium a diferentes cultivares de milho. Semina: Ciências Agrárias, v. 30, n. 1, p. 1123-1134, 2009.

DRANCA, A. C.; HELVIG, E. O.; MACIEL, C. D. de G.; CARBONARI, C. A.; VELINI, E. D. Associações de herbicidas com fertilizante foliar e regulador vegetal em soja. Pesquisa Aplicada \& Agrotecnologia, v. 11, n. 3, p. 69-80, 2018.

DOI: 10.5935/PAeT.V11.N3.07.

ESPANHOL, M. Seletividade de nicosulfuron isolado e em mistura com atrazine para trinta híbridos comerciais de milho. 2009. 44 f. Dissertação (Mestrado) - Faculdade de Ciências Agrárias e Veterinárias, Universidade Estadual Paulista, Jaboticabal, 2009.

GIOVANELLI, B. F.; SILVA, A. F. M.; ALBRECHT JÚNIOR, A. P.; AIELLO, L. H. F.; GHIRARDELLO, G. A.; ALBRECHT, L. P.; VICTORIA FILHO, R. Selectivity of herbicides applied separately or in combination in the post emergence of RR2 maize. Brazilian Journal of Agriculture, v. 93, n. 1, p. 47-57, 2018.

HAMZA, B.; SUGGARS, A. Biostimulants: myths and realities. Turfgrass Trends, v. 10, p. 6-10, 2001.

LÓPEZ-OVEJERO, R. F.; FANCELLI, A. L.; DOURADONETO, D.; GARCÍA Y GARCÍA, A.; CHRISTOFFOLETI, P. J. Seletividade de herbicidas para a cultura do milho (Zea mays) aplicados em diferentes estádios fenológicos da cultura. Planta Daninha, v. 21, n. 3, p. 413-419, 2003. DOI: $10.1590 / \mathrm{S} 0100-83582003000300009$.

MACIEL, C. D. G.; OLIVEIRA NETO, A. M.; GUERRA, N.; JUSTIANO, W. Eficiência e qualidade da aplicação de misturas em tanque com adjuvantes na dessecação de corda-de-viola. Engenharia Agrícola, v. 31, n. 4, p. 704715, 2011. DOI: 10.1590/S0100-69162011000400009.
MACHADO, M. S.; FERREIRA, L. R.; PAULA, J. L.; PEREIRA, G. A. M.; GONÇALVES, V. A. Use of liquid fertilizer to reduce the phytotoxic effects of glyphosate on eucalyptus. Revista Caatinga, v. 30, n. 3, p. 730-737, 2017 a.

DOI: 10.1590/1983-21252017v30n321rc.

MACHADO, M. S.; FERREIRA, L. R.; PEREIRA, G. A. M.; PAUlA, J. L.; PAIXÃO, G. P.; FREITAS, P. H. M. Fertiactyl Pós ${ }^{\circledR}$ como protetor do eucalipto submetido a aplicação de glyphosate. Revista Brasileira de Ciências Agrárias, v. 12, n. 2, p. 194-201, 2017 b.

DOI: 10.5039 /agraria.v12i2a5425.

NARDI, S.; PIZZEGHELLO, D.; SCHIAVON, M.; ERTANI, A. Plant biostimulants: physiological responses induced by protein hydrolyzed-based products and humic substances in plant metabolism. Scientia Agricola, v. 73, n. 1, p. 18-23, 2016. DOI: 10.1590/0103-9016-2015-0006.

NICOLAI, M.; CARVALO, S. J. P. de; LÓPEZ-OVEREJO, R. F.; CHRISTOFFOLETI, P. J. Aplicação conjunta de herbicidas e inseticidas na cultura do milho. Bragantia, v. 65, n. 3, p. 413-420, 2006.

DOI: $10.1590 / \mathrm{s} 0006-87052006000300007$.

OLIVEIRA NETO, A. M.; OLIVEIRA JÚNIOR, R. S.; CONSTANTIN, J.; ALONSO, D. G.; RAIMONDI, M. A.; SANTOS, G.; GEMELLI, A. Modalidades de aplicação e associações de herbicidas no controle de plantas daninhas em milho em espaçamento convencional e reduzido. Semina: Ciências Agrárias, v. 32, n. 1, p. 81-92, 2011. DOI: $10.5433 / 1679-0359.2011 v 32 n 1 p 81$.

PEEL, M. C.; FINLAYSON, B. L.; MCMAHON, T. A. Updated world map of the Köppen-Geiger climate classification. Hydrology Earth System Sciences, v. 11, n. 5, p. 1633-1644, 2007.

DOI: $10.5194 /$ hess-11-1633-2007.

PARKER, R. G.; YORK, A. C.; JORDAN, D. L. Weed control in glyphosate-resistant corn as affected by preemergence herbicide and timing of postemergence herbicide application. Weed Technology, v. 20, n. 3, p. 564-570, 2006. DOI: 10.1614/WT-04-288R.1. 
PORTUGAL, L. V. Fitotoxicidade de herbicidas pósemergentes em híbridos de milho. 2013. 51 f. Dissertação (Mestrado em Sistemas de Produção na Agropecuária) Universidade José do Rosário Vellano, Alfenas, 2013.

RIZZARDI, M. A.; ZANATTA, F. S.; LAMB, T. D.; JOHANN, L. B. Controle de plantas daninhas em milho em função de épocas de aplicação de nitrogênio. Planta Daninha, v. 26, n. 1, p. 113-121, 2008.

DOI: $10.1590 / \mathrm{S} 0100-83582008000100012$.

SANTOS, A. C. M.; SOUZA, M. A. S.; FREITAS, G. A.; SANTOS, P. S. S.; SILVA, R. R. Substância húmica na redução da fitotoxicidade dos herbicidas Roundup Ready + Lactofen na cultura da soja. Tecnologia \& Ciência Agropecuária, v. 9, n. 3, p. 35-41, 2015.

SANTOS, H. G. dos; JACOMINE, P. K. T.; ANJOS, L. H. C. dos; OLIVEIRA, V. A. de; LUMBRERAS, J. F.; COELHO, M. R.; ALMEIDA, J. A. de; CUNHA, T. J. F.; OLIVEIRA, J. B. de. Sistema brasileiro de classificação de solos. 3. ed. rev. e ampl. Brasília, DF: Embrapa, 2013a. $353 \mathrm{p}$.

SANTOS, V. M.; MELO, A. V.; CARDOSO, D. P.; GONÇALVES,A.H.; VARANDA, M.A. F.; TAUBINGER, M. Uso de bioestimulantes no crescimento de plantas de Zea mays L. Revista Brasileira de Milho e Sorgo, v. 12, n. 3, p. 307-318, 2013b.

DOI: 10.18512/1980-6477/rbms.v12n3p307-318.
SILVA, J. A. A. da; COELHO, A. M.; GAZZIERO, D. L. P.; VARGAS, L.; KARAM, D. Aminoácidos como recuperadores de plantas de milho intoxicadas por nicosulfuron. In: CONGRESSO NACIONAL DE MILHO E SORGO, 28.; SIMPÓSIO BRASILEIRO SOBRE A LAGARTA DO CARTUCHO, 4., 2010, Goiânia. Potencialidades, desafios e sustentabilidade: resumos expandidos... Sete Lagoas: ABMS, 2010. 1 CD-ROM

SPADER, V.; VIDAL, R. A. Seletividade e dose de injúria econômica de nicosulfuron aplicado em diferentes estádios de desenvolvimento da cultura do milho. Ciência Rural, v. 31, n. 6, p. 929-934, 2001.

DOI: $10.1590 / \mathrm{S} 0103-84782001000600001$.

TERRA, M. A.; MARTINS, D.; COSTA, N. V.; MARCHI, S. R. Avaliação de pontas e taxas de aplicação na deposição de calda no milho e em plantas daninhas. Bioscience Journal, v. 30, n. 6, p. 1661-1670, 2014.

TIMAC AGRO. Dossiê Fertiactyl Pós: dossiê técnicocientífico. Porto Alegre, 2014. 92 p.

VELINI, E. D.; OSIPE, R.; GAZZIERO, D. L. P. (Coord.). Procedimentos para instalação, avaliação e análise de experimentos com herbicidas. Londrina: SBCP, 1995. $42 \mathrm{p}$. 